\title{
El crédito productivo y su incidencia en la producción agrícola del Ecuador
}

\author{
Productive credit and its impact on agricultural \\ production in Ecuador \\ O crédito produtivo e sua incidência na produção \\ agrícola do Equador
}

\section{Lenin Chagerben}

Universidad de Guayaquil (Ecuador)

lenin.chagerbensa@ug.edu.ec

\section{Noemí Moreno}

Universidad de Guayaquil (Ecuador)

noemoga@hotmail.com

\section{Werner Chagerben}

Universidad de Guayaquil (Ecuador)

wsalinas.chagerben@gmail.com

DOI: https://doi.org/10.32719/25506641.2019.6.1

Fecha de presentación: 8 de junio de 2019 • Fecha de aceptación: 15 de agosto de 2019

Artículo de investigación

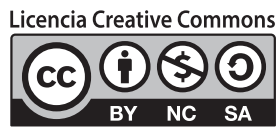




\section{Resumen}

El sector agrícola es muy relevante en la economía ecuatoriana, debido al gran aporte que tiene como fuente de empleo y por su contribución al PIB nacional. Por esta razón, las políticas y estrategias públicas están enfocadas hacia el crecimiento sostenido de la producción agrícola. Un instrumento de dichas políticas ha sido el otorgamiento de créditos productivos. Es importante resaltar que el sector privado registra una relevante contribución respecto a estos créditos, siendo esta mayor que lo registrado en el sector público. El objetivo de esta investigación fue explorar la evolución del crédito productivo agrícola y la incidencia que ha tenido en la producción agrícola, delimitados por zonas de planificación, período 2005-2018. La hipótesis planteada fue: el aumento en el nivel de créditos concedidos, tanto público como privado, coadyuva al crecimiento de la producción agrícola; misma que no fue aceptada según la evidencia analizada.

Palabras clave: Sector agrícola, crédito productivo, modelamiento econométrico.

JEL: C51 Construcción de modelos y estimación; I38 Política pública.

\section{Abstract}

The agricultural sector is very relevant in the Ecuadorian economy, due to its great contribution as a source of employment as well as to the national GDP. Thus, public policies and strategies are focused on the agricultural production sustained growth. An instrument of these policies has been the granting of productive credits - it is important to emphasize that the private sector registers a significant contribution regarding these credits, being this, greater than the one registered in the public sector. The objective of this research was to explore the evolution of agricultural productive credit and its impact on agricultural production, delimited by planning areas, period 2005-2018. The hypothesis was: the increasing in the level of granted credits, both public and private, contributes to agricultural production growth; which was not accepted according to the analyzed evidence.

Keywords: Agricultural sector, productive credit, econometric modeling. JEL: C51, I38.

\section{Resumo}

O setor agrícola é muito relevante na economia equatoriana, devido ao grande apoio que possui como fonte de emprego e por sua contribuição ao PIB nacional. Por essa razão, as políticas e estratégias públicas estão enfocadas no crescimento sustentável da produção agrícola. Um instrumento destas políticas tem sido a outorga de créditos produtivos - é importante ressaltar que o setor privado registra uma relevante contribuição com respeito a esses créditos, sendo esta, maior que o registrado no setor público. O objetivo desta investigação foi explorar a evolução do crédito produtivo agrícola e a incidência que teve na produção agrícola, delimitados por zonas de planejamento, período 2005-2018. A hipótese apresentada foi: o aumento no nível de créditos concedidos, tanto público como privado, contribuiu para o crescimento da produção agrícola; a mesma que não foi aceita segundo a evidência analisada.

Palavras-chave: Setor agrícola, crédito produtivo, modelamento econométrica.

JEL: C51 Construção de modelos e estimativa; I38 Política pública. 


\section{Introducción}

cuador, a través de su historia, se ha caracterizado por poseer una economía basada en la explotación de los recursos naturales. En términos económicos, el sector primario ha sido sustancial para el impulso de un crecimiento y desarrollo económico sostenible. En el siglo anterior, la economía ecuatoriana tuvo varios booms productivos, tales como: el bananero, el cacaotero y el petrolero, que auguraban grandes beneficios para el desarrollo de la sociedad; pero resultó lo contrario: la década pérdida de 1980. A excepción del boom petrolero, los otros dos auges económicos surgieron del sector agrícola; y desde aquella época, este sector ha sido muy importante para la economía y desarrollo de la sociedad ecuatoriana.

Debido al impacto del sector agrícola en la economía ecuatoriana, las políticas y estrategias de desarrollo han sido direccionadas hacia el impulso, fortalecimiento y diversificación de aquel sector económico. Estas políticas y estrategias fueron impulsadas por la Secretaría Nacional de Planificación y Desarrollo (SENPLADES) en el período de Correa y Moreno, de forma similar en el gobierno de Moreno, con la única diferencia de que en el período posterior se eliminó dicha Secretaría. ${ }^{1}$ El sector agrícola, según cifras macroeconómicas del Instituto Nacional de Estadística y Censos (INEC 2019), mantiene su estructura productiva, social y económica, y según la misma institución, el empleo adecuado en este sector pasó del 13,2 al 10,9\% entre diciembre de 2007 y diciembre de 2018. Cabe destacar que el sector agrícola es uno de los que más absorbe a la población económicamente activa (PEA). En este período de tiempo, el empleo adecuado en el sector agrícola se ha destruido; es decir, aquellas políticas no han cambiado la estructura socioeconómica y productiva del país.

1. Desde el 13 de mayo de 2019 la SENPLADES fue remplazada por Planifica Ecuador. 
Es claro que el nivel de empleo es un indicador que involucra tanto el aspecto social como el económico; asimismo, está sujeto al crecimiento económico, ya sea a nivel país (macro) como a nivel sector agrícola (micro); en efecto, incrementar la producción agrícola influirá en el crecimiento del empleo en aquel sector y en la economía nacional, dada su participación. En contraste, el estancamiento productivo o declive del sector tendrá un efecto negativo en la creación de nuevos empleos.

La relevancia que posee este sector en la economía del país, como se ha demostrado con el acaparamiento de la PEA y el estancamiento del mismo, amerita que se efectúen investigaciones objetivas que demuestren cuán eficiente han sido las políticas y estrategias que han tomado los diferentes gobiernos centrales. En la presente investigación se busca cuantificar la incidencia que tienen los créditos productivos en la producción agrícola. En contraste con Quinde Rosales, Bucaram Leverone y Quinde Rosales (2018), esta investigación hace uso de las zonas de planificación, lo cual permite observar las variaciones que existen entre aquellas, por lo tanto, le dan un grado de robustez al estudio.

Para llevar a cabo el análisis central de la investigación, se tomó en consideración el modelamiento econométrico utilizando las combinaciones de cortes transversales en el tiempo, también llamado panel de datos (Wooldridge 2010). Pero, antes de realizar el modelo final que estimó la sensibilidad que tiene la producción agrícola dada su ubicación geográfica, se realizó un análisis descriptivo de las variables circunscritas a la investigación.

El objetivo de esta investigación es explorar la evolución del crédito productivo agrícola y la incidencia que este ha tenido en los niveles de producción agrícola, delimitados por zonas de planificación, para el período 2005-2018. La hipótesis que se planteó es: el aumento en los niveles de crédito concedido, tanto público como privado, coadyuva al crecimiento de la producción agrícola.

La presente investigación está compuesta por las siguientes: introducción, revisión literaria, metodología, resultados, discusión de resultados, conclusiones y recomendaciones, referencias bibliográficas y anexos. 


\section{Revisión de literatura}

En Ecuador, la literatura con respecto a la relación existente entre el crédito productivo destinado al sector agrícola y su nivel de producción es relativamente corta, por lo que es importante considerar los estudios elaborados en otros países de América Latina. Las diferencias existentes entre los países latinoamericanos son sustanciales a nivel cultural, sin embargo, a nivel económico la importancia que tiene el sector primario marca una similitud bastante estrecha.

Quinde Rosales, Bucaram Leverone y Quinde Rosales (2018) afirman que el crédito productivo hacia el sector agrícola tomó un gran impulso entre los años 2005-2015; asimismo, mediante la utilización de una regresión múltiple, se demuestra que el crédito otorgado por instituciones públicas tiene poca significancia respecto a los niveles de producción; en contraste, el crédito privado y de otras entidades financieras mantiene una relación inversa con los niveles de producción.

La utilización de regresiones lineales múltiples, tal como se presenta en la investigación de Quinde Rosales, Bucaram Leverone y Quinde Rosales (2018), puede ser inadecuado debido a que podrían existir más variables que afecten a la producción agrícola (Gujarati y Porter 2010). Además, puede darse que en los modelos de regresión lineal no se tome en cuenta los efectos inobservables, tales como las diferencias que existan entre los agentes agrícolas. En el caso de los modelos econométricos de datos de panel, estos podrían tomar en cuenta los efectos inobservables significativos, tanto para la sección transversal (agentes/zonas) como para la serie de tiempo.

Por otro lado, respecto a la importancia que tiene la economía agrícola para el país, Viteri y Tapia (2018) infieren que la economía ecuatoriana, en la actualidad, no se sustenta en el sector agrícola primario, sino en las industrias enmarcadas en el sector de los servicios. El análisis de Viteri y Tapia (2018) está basado en la concentración de la producción nacional, la cual está dada por el Producto Interno Bruto (PIB); en este se comprueba que la producción agrícola solo concentra el 7\%, aproximadamente, del PIB, mientras que un $57 \%$ del PIB está concentrado en actividades relacionadas al servicio. 
El PIB es una medición enfocada directamente a la producción monetizada durante un año, es decir, busca medir el crecimiento económico que tiene una nación a través del tiempo. En contraste, el nivel de empleo adecuado busca medir el nivel de desarrollo que tiene una sociedad, ya que de este dependerá el consumo, ahorro e inversión que tendrá un individuo. Aunque la presente investigación está centrada en la exploración de la relación entre el crédito productivo agrícola y el nivel de producción, es importante identificar la relevancia que tiene el sector agrícola en la nación.

García Navarro (2017) en el estudio realizado en Nicaragua, afirma que existen otras variables que afectan directamente a la producción agrícola, tales como: sobrevaloración cambiaria, poco acceso a fuentes de financiamiento e ínfima capitalización de los productores. La sobrevaloración de una moneda permite que las importaciones de bienes y/o servicios aumenten, ya que sus precios son, superficialmente, más bajos que los internos. Por otro lado, el difícil acceso o restricción a fuentes de financiamiento no les permite a los pequeños y medianos productores capitalizarse, por lo que su capacidad de mantenerse escasea.

En Ecuador, las importaciones podrían ser más baratas debido a shocks externos, lo cual se ha convertido en un talón de Aquiles para la economía doméstica. Algunos de estos shocks externos son la apreciación del dólar y la devaluación de las monedas de los países socios/vecinos, entre los principales. Por otro lado, las exportaciones ecuatorianas (mayoritariamente commodities) podrían verse afectadas debido a la caída de los precios internacionales de los commodities. Este es un problema exógeno que afecta al sector agrícola. Por ende, las políticas de créditos deberían ir enfocadas al fortalecimiento y potenciación de la competitividad de los productores agrícolas. En esta investigación se comprueba qué tan sensible es la producción agrícola al crédito productivo.

Una de las herramientas estadísticas que coadyuva a explorar la relación entre dos o más variables es la correlación de Pearson o Spearman, las cuales están dadas por la distribución que tengan los datos recopilados. Fuentes-Méndez (2005) hace referencia al análisis de correlación para las variables: crédito público y producción. Determina que el grado de asociación lineal entre estas variables fue débil-moderada, y en la mayoría de los casos, inversa. En adición, Fuentes-Méndez (2005) al igual que Quinde Ro- 
sales, Bucaram Leverone y Quinde Rosales (2018), utilizaron las regresiones múltiples, por lo cual podrían haber caído en el problema de estimación y/o sesgo de especificación, además de no tomar en cuenta los efectos inobservables en los agentes y/o el período.

En contraste con Fuentes-Méndez (2005) y Quinde Rosales, Bucaram Leverone y Quinde Rosales (2018), Echavarría, Restrepo, Villamizar y Hernández (2017), en el estudio realizado para el agro en Colombia, llegan a la conclusión de que los diversos tipos de créditos tienen un efecto positivo y significativo sobre el rendimiento (entre 3 y $28 \%$ de la producción). Además, este estudio fue realizado mediante un análisis de Propensity Score Matching, cuya metodología es no experimental, por lo que se trata de un análisis basado, netamente, en los datos observables, utilizando algoritmos.

Se observa que usando los modelos de regresiones lineales múltiples se generan los mismos resultados, mientras que tomando en consideración otra metodología, los resultados son totalmente diferentes. Esta investigación plantea una metodología distinta a las mencionadas anteriormente. En la siguiente sección se describe la metodología que se aplica para, a posteriori, comprobar la hipótesis planteada en la investigación; asimismo, se comparan los resultados obtenidos con los resultados obtenidos en las investigaciones descritas en este apartado.

\section{Metodología}

Dada la naturaleza de la información que brindan las fuentes oficiales del Ecuador, esta ha sido manipulada de tal manera que sirva para los fines de esta investigación; entre las fuentes primarias de información se encuentran: Sistema de Información Pública Agropecuaria (SIPA), INEC y Superintendencia de Bancos.

En el SIPA se encontró información con respecto a la producción agrícola por provincias y zonas de planificación para el período de estudio. Por otro lado, en el INEC se recopiló información respecto al nivel de empleo caracterizado por el sector agrícola; y, por último, en el sistema de información estadística de la Superintendencia de Bancos se identificaron los montos otorgados en créditos. 
Respecto a los niveles de créditos otorgados, ya sea público o privado, estos fueron homologados por subsistema de entidad y por las zonas de planificación. Las herramientas utilizadas para procesar la información fueron Microsoft Excel, y para el modelamiento de la información se utilizó Stata y RStudio. A continuación, se detallan las variables utilizadas en esta investigación.

\section{Variables de estudio}

Las variables de estudio están ligadas directamente al cumplimiento del objetivo y a la comprobación de la hipótesis planteada: nivel de producción agrícola, variable explicada; zona de planificación, variable explicativa; y, por último, el crédito productivo agrícola privado y público, todas variables explicativas. En términos matemáticos se tendría lo siguiente:

Nivel de producción agrícola $=\mathrm{F}$ (Zona de planificación, CredPúblico, CredPrivado)

Es decir que el nivel de producción agrícola estará explicada o en función de la zona de planificación y del crédito productivo agrícola público y privado. Cabe destacar que, a diferencia de Quinde Rosales, Bucaram Leverone y Quinde Rosales (2018), en la modelación econométrica sí se tomaron en cuenta los agentes (zonas) a quienes se les otorgó el crédito. A continuación, se definen cada una de las variables que se consideró para la investigación y se detalla cómo están estratificadas las provincias del país mediante zonas (tabla 1).

\section{Modelo de datos de panel}

El principal insumo para cualquier tipo de modelamiento estadístico, matemático o econométrico son los datos. Debido a esto, se debe tener especial cuidado al momento en que son recopilados y manipulados. Específicamente, los modelos de datos de panel son poco utilizados, ya que no existen muchas fuentes que levanten la información de forma que se puedan elaborar 
Tabla 1

\section{Definición de las variables de estudio}

\begin{tabular}{|l|l|}
\hline \multicolumn{1}{|c|}{ Variables } & \multicolumn{1}{c|}{ Definición } \\
\hline Nivel de producción agrícola & $\begin{array}{l}\text { Producción anual de productos agrícolas medida en } \\
\text { toneladas. }\end{array}$ \\
\hline Zona de planificación & $\begin{array}{l}\text { Agrupación estratégica territorial de las provincias } \\
\text { del Ecuador. }\end{array}$ \\
\hline Crédito productivo agrícola público & $\begin{array}{l}\text { Monto otorgado anualmente por instituciones públi- } \\
\text { cas enfocado a la agricultura y actividades conexas, } \\
\text { agrupado por las zonas de planificación. }\end{array}$ \\
\hline Crédito productivo agrícola privado & $\begin{array}{l}\text { Monto otorgado anualmente por instituciones } \\
\text { privadas enfocado a la agricultura y actividades } \\
\text { conexas, agrupado por las zonas de planificación. }\end{array}$ \\
\hline
\end{tabular}

Nota. Los créditos públicos y privados están estratificados por las zonas de planificación; de la misma forma, el nivel de producción.

Elaboración propia.

los modelos de datos de panel. Entre las fuentes oficiales que proveen información de tipo panel están las siguientes: Organización para la Cooperación y el Desarrollo Económico (OCDE), Panel Study of Income Dynamics (PSID) y la European Community Household Panel (ECHP).

La composición de una base de datos de tipo panel está dada por la combinación de información recopilada a través del tiempo o también llamado times series (períodos), y de información de corte transversal o también llamado cross sectional (empresas, agentes, individuos). En otras palabras, son observaciones de diversos agentes/individuos/empresas a través del tiempo/ período. Los datos se recopilan para cada uno de los individuos $i=1,2,3 \ldots N$, en cada uno de los períodos del tiempo, $t=1,2,3 \ldots T$ (Gujarati y Porter 2010).

El modelamiento a través de datos de panel enriquece cualquier análisis empírico, lo cual no sería posible si se modelara de forma individual, tanto las series de tiempo como los datos de corte transversal (Arellano y Bover 1990). Entre los usos que tienen los modelos de datos de panel o longitudinales está la evaluación del impacto de ciertos eventos o políticas (Wooldridge 2010). Además, Arellano y Bover (1990) destacan que el uso de datos de panel evita problemas de agregación y facilita el seguimiento del comporta- 
Figura 1

\section{División estratégica de Ecuador}

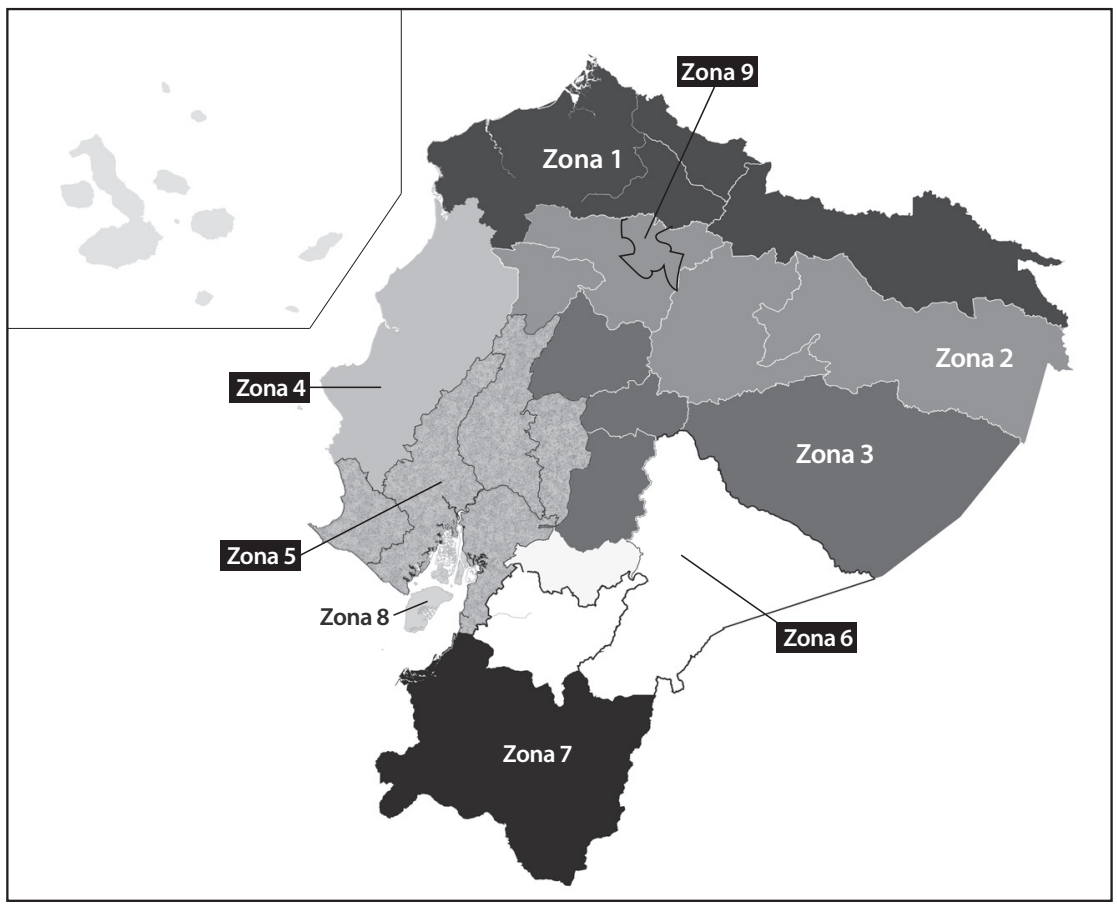

Nota. Para la investigación se utilizaron solo 7 zonas, ya que se consideró a Guayaquil, Durán y Samborondón dentro de la zona 5; y, por otro lado, en la zona 2 se incorporó a Quito.

Fuente: SENPLADES (2019).

miento de cada individuo a través del tiempo, y permite estimar modelos que tienen presentes las diferencias permanentes entre los individuos, aunque estas no sean observables.

Por otro lado, en los datos de panel se debe tener en cuenta el balance que existe entre el número de observaciones, tanto para la serie de tiempo como para los individuos del corte transversal. En efecto, este tipo de datos pueden ser balanceados o no balanceados. Respecto a los datos balanceados, se refiere a aquellos donde cada agente tiene el mismo número de observaciones. En contraste, los datos no balanceados se originan cuando hace falta alguna 
observación para algún agente. En adición, los paneles pueden ser cortos o largos, dependerá del número de agentes y períodos de tiempo; en aquellos donde el número de agente $(i)$ es mayor que el número de períodos $(t)$ se trata de un panel corto; al contrario, el panel de datos largo. Para el modelamiento de datos de panel existen tres tipos que pueden utilizarse, dependerá de las pruebas o contrastes estadísticos que arrojen cada uno de aquellos tipos. A continuación, se ilustran los tipos de modelos de datos de paneles estáticos:

Figura 2

\section{Tipos de modelos de datos de panel estáticos}



Nota. En este caso solo se han mencionado a los modelos de datos de panel estáticos, pero también existen dinámicos, tales como variables instrumentales.

Fuente: Gujarati y Porter (2010).

Elaboración propia. 
En el modelo Pool no se considera la heterogeneidad entre los períodos de tiempo ni entre los agentes. En otras palabras, se trata de una regresión múltiple donde sus coeficientes son estimados mediante los mínimos cuadrados ordinarios (MCO); por otro lado, los efectos fijos (EF) y los efectos aleatorios (EA) sí consideran las diferencias en $i$ y $t$. En adición, la diferencia entre EF y EA radica en que el primero asume los efectos inobservables correlacionados con las variables explicativas del modelo; mientras el segundo es más restrictivo y, por ende, no acepta correlación entre ambas, y de allí se originan valores aleatorios tanto para $i$ como para $t$. La nomenclatura de un modelo de datos de panel en la forma general es la siguiente:

$$
y_{i t}=\alpha+X_{i t} \beta+\mu_{i}+\theta_{t}
$$

Donde $Y$ it es la variable dependiente, en este caso, el nivel de producción agrícola medida en toneladas de cada zona con $i=1,2,3 \ldots 7$, teniendo en cuenta la dimensión periódica, de $t=1,2,3 \ldots 14$. Por otro lado, Xit se trata del vector $k$ variables explicativas. $\mu$ i denota el efecto de la heterogeneidad que es inobservable para cada zona de planificación y constante para los períodos de tiempo. Y, por último, $\theta \mathrm{t}$ es el término de error. El modelamiento de panel diferencia que tales efectos inobservables se comporten como fijos o aleatorios, dependerá de los resultados que arrojen los diversos contrastes estadísticos. A continuación se muestran los diversos contrastes que evalúan la pertinencia para el modelamiento de datos de panel, con estos se sabrá qué tipo de modelo hacer uso (ver tabla 2).

Entonces, en virtud de lo que se manifestó en la parte introductoria de la investigación, con esta se busca explorar la relación, dada por la sensibilidad/elasticidad, entre el nivel de producción agrícola (delimitada por las zonas de planificación) y el crédito productivo otorgado tanto por entidades públicas como privadas. Para estimar la sensibilidad de la variable dependiente respecto a las variables independientes, la ecuación 1 deberá sufrir un cambio. Este cambio se trata de implementar a las variables numéricas del modelo un logaritmo natural (ln); con este cambio, el modelo econométrico quedaría de la siguiente forma:

$$
\ln \left(y_{i t}\right)=\alpha+\beta_{2} \ln X_{1}+\beta_{3} \ln X_{2}+\mu_{\mathrm{i}}+\theta_{\mathrm{t}}
$$


Llevándolo a la investigación, quedaría:

$$
\ln \text { prod }=\alpha+\beta_{2} \operatorname{lnCredPub}+\beta_{3} \ln \text { CredPriv }+\mu_{\mathrm{i}}+\theta_{\mathrm{t}}
$$

La aplicación de logaritmo natural no solo coadyuva a la interpretación de los coeficientes como elasticidades, sino también a transformar los datos de aquellas variables en información estacionaria, es decir, con varianza y media constante. En otras palabras, vuelve homocedástica la serie. Por otro lado, cabe destacar que la variable zona es una variable dicotómica y que al modelizar esta se introduce en el resultado del modelamiento final. En el apartado posterior se presentan los resultados empíricos de la investigación, empezando con estadísticas descriptivas de la información recopilada de las diversas fuentes de información oficiales.

Tabla 2

\section{Contrastación entre modelos de datos de panel}

\begin{tabular}{|c|c|c|c|}
\hline Comparación & Contrastes/Prueba & Hipótesis & Decisión \\
\hline Pool vs. EF & Prueba F (1920) & $\begin{array}{c}H_{0}: \mu_{1}=\mu_{2}=\mu_{3} \\
H_{1}: \mu_{1} \neq \mu_{2} \neq \mu_{3}\end{array}$ & $\begin{array}{c}\text { Rechazo } \\
\text { Elección EF }\end{array}$ \\
\hline EAvs. Pool & Breusch-Pagan (1979) & $\begin{array}{c}H_{0}: \operatorname{Cov}\left(\mu_{1}\right)=0 \\
H_{1}: \operatorname{Cov}\left(\mu_{1}\right) \neq 0\end{array}$ & $\begin{array}{c}\text { Rechazo } \\
\text { Elección EA }\end{array}$ \\
\hline EF vs. EA & Test Hausman (1978) & $\begin{array}{c}H_{0}: \operatorname{Corr}\left(\mathrm{X}_{\mathrm{it}}, \mu_{1}\right)=0 \\
H_{1}: \operatorname{Corr}\left(\mathrm{X}_{\mathrm{it}}, \mu_{1}\right) \neq 0\end{array}$ & $\begin{array}{c}\text { Rechazo } \\
\text { Elección EF }\end{array}$ \\
\hline
\end{tabular}

Nota. Para aceptar o rechazar la Hipótesis Nula $\left(H_{0}\right)$ se debe considerar la probabilidad del estadístico que se esté usando. Si p<0,05 no existe evidencia suficiente para aceptar la $H_{0}$.

Elaboración propia.

\section{Resultados}

El análisis estadístico descriptivo es primordial previo a cualquier modelamiento, ya sea econométrico o estadístico. Esto permite tomar las correcciones a tiempo, en caso de que los datos recopilados muestren alguna anormalidad, como: datos atípicos, distribución no normal, datos ausentes, entre otros. Cabe destacar que, al tratarse de datos longitudinales o datos de 
panel, las estadísticas descriptivas que se muestran a continuación tienen una especial interpretación, la cual será minuciosamente detallada.

Tabla 3

Estadísticas descriptivas de las variables de estudio

\begin{tabular}{|l|l|l|r|r|r|c|}
\hline \multicolumn{2}{|c|}{ Variables } & Media & $\begin{array}{c}\text { Desviación } \\
\text { estándar }\end{array}$ & Mínimo & Máximo & Observaciones \\
\hline \multirow{4}{*}{ Producción } & General & $3.099,16$ & $4.490,66$ & 194,86 & $15.970,61$ & $\mathrm{~N}=98$ \\
\cline { 2 - 7 } & Entre & & $4.768,60$ & 429,62 & $13.726,37$ & $\mathrm{n}=7$ \\
\cline { 2 - 7 } & Dentro & & 688,51 & 618,10 & $5.343,39$ & $\mathrm{~T}=14$ \\
\hline \multirow{3}{*}{$\begin{array}{l}\text { Crédito } \\
\text { público }\end{array}$} & General & $\$ 19.715,49$ & $\$ 23.687,66$ & $\$ 1.898,10$ & $\$ 149.447,20$ & $\mathrm{~N}=98$ \\
\cline { 2 - 7 } & Entre & & $\$ 20.765,64$ & $\$ 3.747,04$ & $\$ 65.153,05$ & $\mathrm{n}=7$ \\
\cline { 2 - 7 } & Dentro & & $\$ 13.699,80$ & $-\$ 25.141,60$ & $\$ 104.009,70$ & $\mathrm{~T}=14$ \\
\hline \multirow{3}{*}{$\begin{array}{l}\text { Crédito } \\
\text { privado }\end{array}$} & General & $\$ 66.421,25$ & $\$ 76.195,59$ & $\$ 103,27$ & $\$ 315.283,30$ & $\mathrm{~N}=98$ \\
\cline { 2 - 7 } & Entre & & $\$ 60.261,93$ & $\$ 22.752,37$ & $\$ 167.590,20$ & $\mathrm{n}=7$ \\
\cline { 2 - 7 } & Dentro & & $\$ 51.584,53$ & $-\$ 86.912,20$ & $\$ 214.114,30$ & $\mathrm{~T}=14$ \\
\hline
\end{tabular}

Nota. Las cantidades proporcionadas en la tabla se encuentran en miles (dólares y toneladas, según sea el caso). Fuente: El sumario fue calculado a partir de los datos obtenidos en la Superintendencia de Bancos (2019) y el Ministerio de Agricultura, Ganadería, Acuacultura y Pesca (2019).

Elaboración propia.

En la tabla 3 se muestran las estadísticas descriptivas básicas, las cuales dan una leve y breve percepción de cómo está la situación referente a las variables objeto de estudio. Por otro lado, es observable que el promedio anual de la producción de todas las zonas de planificación durante el período 2005-2018 alcanzó una cantidad de 3 millones de toneladas, mientras que su desviación estándar fue de 4,5 millones; pero, es importante destacar que existe una diferenciación bien marcada entre el máximo y mínimo, ya que existe una zona que en un año específico tuvo una producción extraordinaria; mientras que hay otra que produjo una cantidad ínfima. Esta gran brecha entre el máximo y el mínimo incide a favor de una media engañosa. Estas heterogeneidades entre agentes serán absorbidas por el modelo que se demuestra en los próximos apartados. Al tratarse de una base de datos de panel, es prioridad calcularse las 
estadísticas entre los agentes y dentro de los períodos establecidos. Esto está denotado en las características entre (para las zonas) y dentro (para los años), así también para las otras variables, y no solo para la producción.

En la producción es observable que la mayor diferencia se encuentra entre los agentes, es decir que existe una zona que prevalece ante las demás. Mientras que dentro del período de estudio se observa que sí existe una diferencia, pero no en la misma magnitud que entre las zonas de planificación. Con respecto a los montos otorgados por instituciones públicas, en términos generales, el promedio anual alcanzó un valor de USD 20 millones. De forma similar a la producción, este promedio anual podría estar sesgado por la brecha existente entre el mínimo y máximo que existe en aquellos montos. Por último, los montos otorgados por las instituciones privadas presentan características similares referentes al mínimo y al máximo; pero tiene una marcada diferencia respecto al promedio anual de aquellos montos, ya que este alcanzó un valor de USD 66 millones, $247 \%$ por encima de lo otorgado de las instituciones públicas.

Son notorias las diferencias o heterogeneidades que existen tanto entre zonas de planificación y dentro del período tomado para esta investigación. Por lo tanto, se deben considerar dichas características de los datos en el modelo final que estima las elasticidades producción/crédito público y producción/crédito privado. En la página siguiente se demuestran mediante gráficas las heterogeneidades.

La figura 3 ratifica lo demostrado en las estadísticas descriptivas, pero en esta se demuestra, específicamente, en qué zona es la que produce dicha variación o brecha tan elevada. La zona 5 de planificación es la que supera notablemente en el nivel de producción agrícola a nivel nacional a las demás zonas; debido a esto se puede inferir que es la que más demanda créditos, lo cual se ratifica con las figuras de la página siguiente. Por otro lado, la heterogeneidad dentro del período 2005-2018 también es notoria, pero existe una singularidad en comparación con las zonas, y se trata de que existen varios outliers o valores atípicos y, a su vez, muchos valores por debajo de la media, por lo que será necesario linealizar la variable producción para alcanzar una estacionalidad débil y a su vez estimar coeficientes robustos en el modelo final.

Por otro lado, respecto a la distribución en las diferentes zonas de los montos otorgados, tanto por las instituciones privadas y públicas, mantienen 
Figura 3

Heterogeneidad zonas-períodos
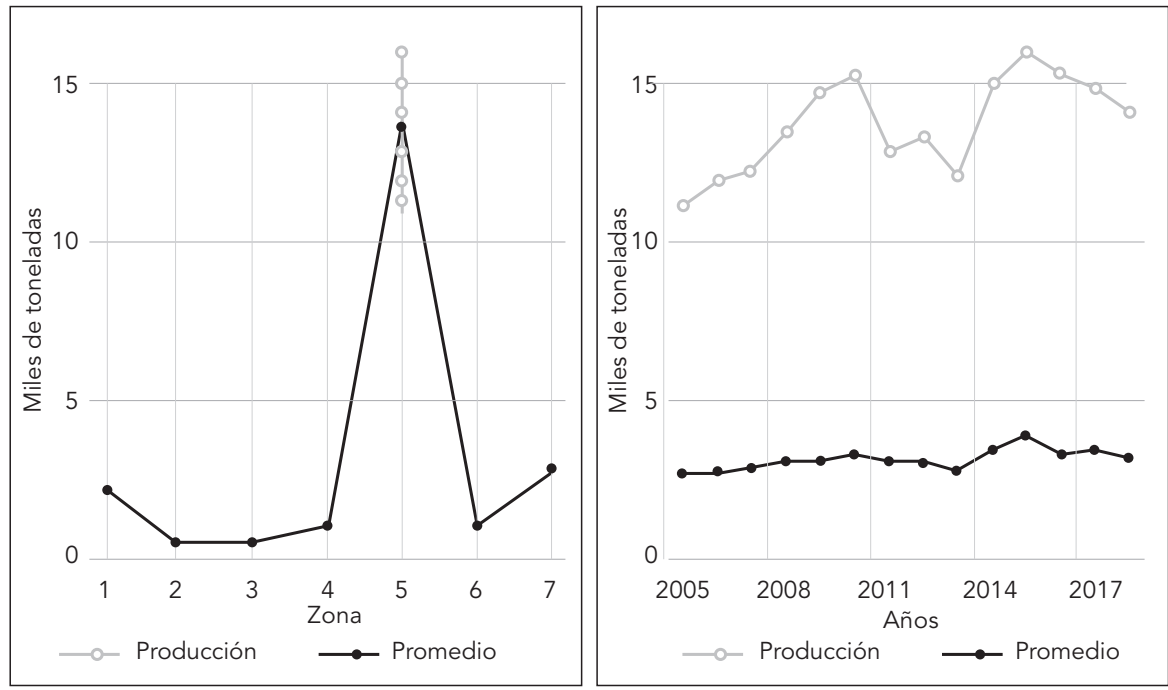

Fuente: SIPA (2019).

un comportamiento similar a lo generado a nivel de producción (figura 4).

El comportamiento que ha tenido el crédito productivo público en las zonas de planificación es similar al que tienen los niveles de producción, es decir, la zona 5 mantiene una brecha bastante amplia respecto a las demás zonas. Mientras que el comportamiento desde la perspectiva de los años, en 2014 se da un ligero despunte frente a los demás años, pero es importante destacar que los créditos desde el sector público, en promedio, no ha superado los USD 20 millones, y estos, a su vez, han sido dirigidos en su mayoría hacia las provincias de la zona 5 del país (figura 5).

En contraste con el crédito productivo agrícola público, el crédito privado mantiene una brecha bastante amplia respecto al nivel de monto que ha otorgado hacia aquel rubro. Además, respecto al otorgamiento por zonas, también se diferencia del sector público, ya que la zona 2 y las demás zonas (exceptuando la zona 5) tienen una participación tibia en el acceso al financiamiento externo. Añadiendo, el comportamiento anual del crédito privado se ha visto mermado durante los últimos tres años; sin embargo, los años 
Figura 4

\section{Comportamiento del crédito público por zonas y tiempo}
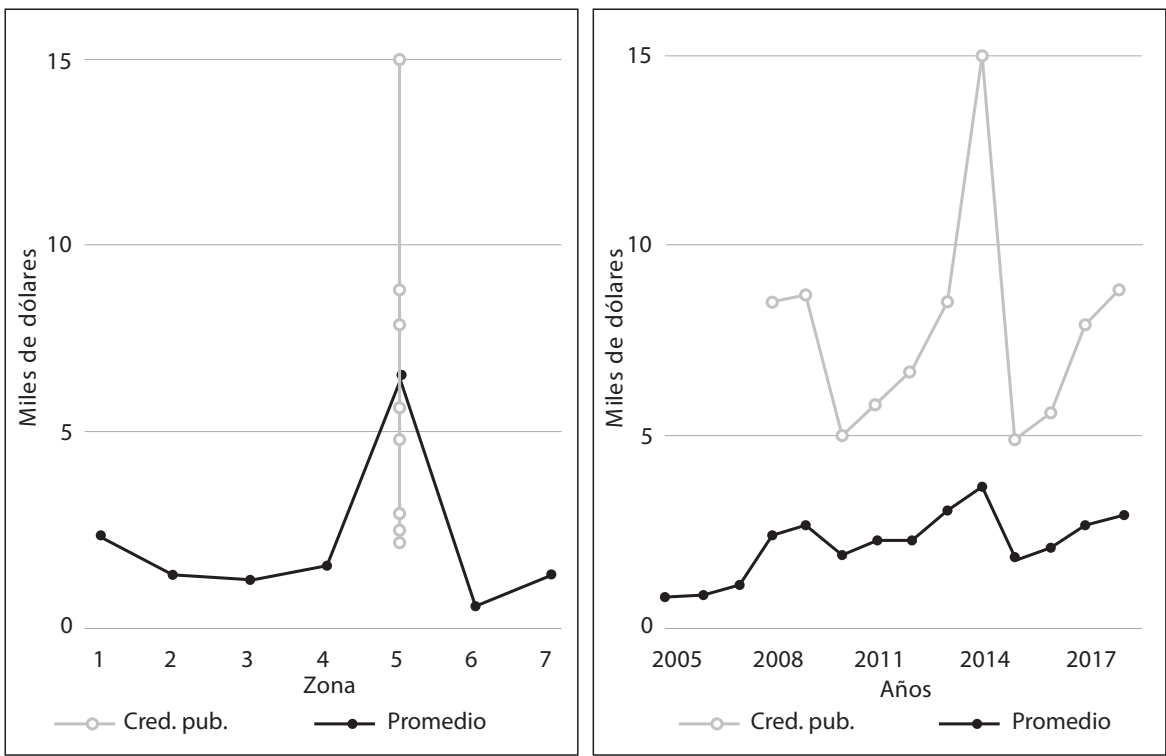

Fuente: Superintendencia de Bancos (2019).

anteriores han otorgado un volumen de crédito bastante amplio respecto al volumen entregado por las instituciones públicas.

Con base en esta descripción del comportamiento de las variables objeto de estudio, grosso modo, se puede inferir que existe una mala focalización del crédito, ya que, tal como se observa en las figuras 3, 4 y 5, la zona 5 concentra gran parte del volumen total de crédito, tanto privado como público. Esta concentración, gráficamente, se refleja en el nivel de producción de dicha zona, marcando una amplia brecha con las demás zonas de planificación. Por otro lado, las instituciones privadas son las que más aportan volumen de crédito hacia el sector agrícola, aunque van disminuyendo en los últimos tres años.

Al identificar las heterogeneidades existentes en las variables a modelar, se procedió a linealizar dichas variables con la finalidad de que tengan una varianza y una media relativamente constante, también conocido como estacionariedad débil. Por el cual, fue suficiente aplicar el logaritmo natural a 
Figura 5

\section{Comportamiento del crédito privado por zonas y tiempo}


Fuente: Superintendencia de Bancos (2019).

aquellas variables. Una vez aplicado el logaritmo natural a las variables se procedió a modelar, haciendo pruebas con los tipos de modelos de datos de panel y aplicando los diversos contrastes con la finalidad de seleccionar al que mejor se adapta a la base de datos recopilada.

En la tabla 4 se muestran los resultados obtenidos aplicando los diferentes tipos de modelos econométricos con base en un panel de datos. La tabla muestra los resultados obtenidos a partir de la ecuación 4 procesada en el programa Stata. Como se menciona en la nota de la tabla 4, los asteriscos indican el nivel de significancia de cada uno de los parámetros resultantes. Una similitud entre todos los modelos plasmados es que la variable que contiene el volumen de crédito otorgado por las instituciones del sector privado no es significativa, es decir, que no explica los cambios en el nivel de producción agrícola. En términos estadísticos, el valor beta $(\beta)$ de dicha variable es igual a cero, por ende, no explica nada. 
Tabla 4

Resultados de los modelos de datos de panel

\begin{tabular}{|c|c|c|c|c|}
\hline Variables & Pool dummy & Pool & Efectos fijos (EF) & Efectos aleatorios (EA) \\
\hline InCredPub & $0,1054^{*}$ & $0,7410^{\star * *}$ & $0,1054^{*}$ & $0,1228^{*}$ \\
\hline InCredPriv & $-0,0115$ & $-0,0194$ & 0,0115 & $-0,0098$ \\
\hline Zona 2 & $-1,6070^{*}$ & & & \\
\hline Zona 3 & $-1,4541^{* * *}$ & & & \\
\hline Zona 4 & $-0,8640^{* * *}$ & & & \\
\hline Zona 5 & $1,7290^{\star \star *}$ & & & \\
\hline Zona 6 & $-0,6406^{* * *}$ & & & \\
\hline Zona 7 & $0,2601^{*}$ & & & \\
\hline Constante & $13,0322^{* * *}$ & 2,4339 & $12,6641^{* * *}$ & $12,3520^{* * *}$ \\
\hline $\mathrm{N}$ & 98 & 98 & 98 & 98 \\
\hline$R^{\wedge} 2$ & 0,9366 & 0,3791 & 0,0492 & \\
\hline $\mathrm{R}^{\wedge} 2$ Ajustado & 0,9309 & 0,3661 & $-0,0362$ & \\
\hline
\end{tabular}

Nota. Dummy se refiere a la creación de una variable dicotómica.

Leyenda: * $p<0,05 ;{ }^{* *} p<0,01 ;{ }^{* * *} p<0,001$.

Elaboración propia.

En contraste, la variable que contiene el volumen de crédito que han otorgado las instituciones públicas hacia el sector agrícola en todos los modelos da como una variable significativa, lo que indica que esta variable explica el comportamiento del nivel de producción agrícola. La diferencia que resalta entre los modelos respecto a esta variable es el nivel de significancia, ya que en el modelo Pool esta es significativa con un 99,99\% de confianza; mientras que en los demás modelos solo arroja un $95 \%$ de confianza.

Entonces, según estos resultados, la única variable que explica y a la que es sensible, aunque en una escala bastante baja (casi nula), es el nivel de producción agrícola con relación al volumen de crédito que han otorgado las instituciones públicas; del volumen proporcionado por instituciones privadas, debido a la evidencia estadística, no se puede realizar una inferencia. Es importante destacar que, grosso modo, la estratificación de las provincias en zonas estratégicas tiene gran relevancia en los cambios en los niveles de producción. En adición, los resultados mostrados en la tabla 4 solo permiten te- 
ner una idea básica de la relación existente entre las variables independientes con la dependiente, ya que estos modelos deberán pasar un riguroso análisis estadístico para, finalmente, llegar a las conclusiones adecuadas.

\section{Tabla 5}

Resultado del mejor modelo de datos de panel (efectos aleatorios)

\begin{tabular}{|c|c|c|c|r|}
\hline Variables & Estimación & Error estándar & Valor $\mathbf{t}$ & $\operatorname{Pr}(>|\mathrm{t}|)$ \\
\hline Constante & 12,3466 & 1,0866 & 11,3623 & $<2 \mathrm{e}-16^{* * *}$ \\
\hline InCredPub & 0,1230 & 0,0559 & 2,2009 & $0,03017^{*}$ \\
\hline InCredPriv & $-0,0097$ & 0,0216 & $-0,4500$ & 0,6538 \\
\hline
\end{tabular}

Nota. No se observan los valores $\mathrm{R}$ debido a las correcciones que se hizo al modelo para que cumpla con todas las pruebas estadísticas.

Leyenda: * $p<0,05 ;{ }^{* *} p<0,01 ;{ }^{* * *} p<0,001$.

Elaboración propia.

Finalmente, luego de un exhaustivo examen de cada uno de los modelos presentados en la tabla 4, se llegó a obtener el modelo que mejor se adaptó a los datos que se recopilaron en el transcurso de la investigación. Las pruebas estadísticas que se realizaron se encuentran en los anexos de la investigación, en los que se puede observar el proceso de depuramiento de los modelos. Comparando las tablas 4 y 5, no existe una gran diferencia respecto al modelo de efectos aleatorios; sin embargo, es necesario acudir a las pruebas para brindar mayor robustez al estudio. El modelo de efectos aleatorios ratifica los resultados ya analizados, es decir que el nivel de producción agrícola es relativamente sensible a los cambios que se da en el volumen de crédito que aportan las instituciones públicas; lo opuesto sucede con el crédito aportado desde el sector privado. En la siguiente sección se contrastarán los resultados obtenidos en esta investigación con las indagaciones que guardan similitud.

\section{Discusión de resultados}

En virtud de lo señalado, el sector agrícola se desenvuelve en un importante rol de la economía ecuatoriana, de allí la importancia de las elabora- 
ciones de indagaciones que aporten con diagnósticos y posibles soluciones a aquellos problemas que acarrea el sector. Los resultados obtenidos en esta investigación están sujetos a una metodología que recoge los efectos entre las zonas de planificación y los períodos de estudio; por esta razón, es relevante realizar un contraste con los resultados que obtuvieron otras investigaciones que guardan similitud en torno al campo que se ha desarrollado en esta.

El estudio realizado por Quinde Rosales, Bucaram Leverone y Quinde Rosales (2018) aplicando un modelo de regresión lineal múltiple, concluye que el volumen de crédito público mantiene una relación poco significativa respecto al nivel de producción, mientras que el crédito privado mantiene una relación negativa. En efecto, según los resultados que se obtuvo en la presente investigación, en contraste con Quinde Rosales, Bucaram Leverone y Quinde Rosales (2018), el volumen del crédito público es significativo a los cambios que presentan los niveles de la producción agrícola. La significancia de una variable no está sujeta al valor per se del parámetro/estimador obtenido, sino al valor del estadístico $t$. Por otro lado, el estimador obtenido en esta investigación 0,1230 indica una relación cuasi perfectamente inelástica entre producción agrícola y crédito productivo público, es decir que los cambios en el crédito público tienen una leve, casi nula, incidencia en la producción.

Respecto al efecto que tiene el crédito productivo privado en la producción agrícola, el presente estudio coincide con lo obtenido por Quinde Rosales, Bucaram Leverone y Quinde Rosales (2018), ya que en ambas metodologías el signo del estimador adherente a dicha variable resultó negativo; sin embargo, aquella variable no tiene significancia alguna, es decir, no explica el comportamiento de la producción dado que el valor del $\beta$ es igual a cero.

Echavarría, Villamizar, Restrepo y Hernández (2017) en su investigación en el agro colombiano concluyen que los diversos tipos de créditos inciden de forma positiva y significativa sobre el rendimiento del agro entre un $3 \mathrm{y}$ $28 \%$. El rendimiento está ligado directamente con los niveles de producción, por ende, el presente estudio se contrapone medianamente, ya que si bien el efecto del crédito público es positivo y significativo, este no guarda la magnitud que se observa en el agro colombiano.

Por otro lado, García Navarro (2017) en el estudio realizado en Nicaragua concluye que el poco acceso a fuentes de financiamiento externas no per- 
mite capitalizar al pequeño y mediano productor, por lo que no pueden hacer frente a innovaciones tecnológicas que coadyuven al desarrollo agrícola. En Ecuador es notable el volumen de crédito que se expande en el sector agríco-

la, pero según los resultados obtenidos en el modelo econométrico, estos no reflejan un mayor impacto en la producción agrícola. Además, se mostró en la sección anterior que dichos montos de créditos están concentrados en una sola zona, por lo que las demás zonas, seguramente, buscan otras fuentes de financiamiento que les permita desarrollarse en el campo ecuatoriano.

En general, los resultados obtenidos en esta investigación han coincidido, ligeramente, con otro estudio realizado en el país en años anteriores; se marca una diferencia, ya que para esta investigación se consideró la delimitación por zonas de planificación, la cual resultó que tiene una incidencia significativa en el comportamiento de la producción agrícola. Por otro lado, se marca una diferencia en el grado de incidencia que tiene el crédito en el agro colombiano frente al ecuatoriano. Esto puede dar origen a nuevas investigaciones que enmarquen las diferenciaciones en el modelo de otorgamiento de créditos en ambos países.

\section{Conclusiones}

El sector agrícola primario para el país representó, en 2017, aproximadamente un $6,04 \%$ del PIB real, que cayó en un $0,25 \%$ respecto al PIB de 2007; sin embargo, del total de los empleos adecuados, el sector agrícola absorbió el 10,80\% (marzo de 2018) por encima de sectores como: construcción, servicios financieros, administraciones públicas, entre otros. El empleo adecuado en este sector aumentó en $0,40 \%$ respecto al mismo mes del año anterior. Entre todas las actividades económicas del país, el sector ocupa el cuarto lugar en generación de empleo adecuado.

Las instituciones financieras privadas han otorgado un volumen de crédito mucho mayor al brindado por instituciones financieras del sector público. Estos créditos productivos han estado concentrados durante el período 20052015. Aproximadamente, el 47,21\% del volumen de crédito otorgado por las instituciones públicas tuvo como destino la zona 5; mientras que el 52,79\% fue para el resto de las zonas de planificación. Por otro lado, menos concen- 
trado que el destino de los créditos públicos, el 36,04\% del volumen de crédito privado fue asignado a la zona 5; mientras que el 63,96\% se asignó para el resto de las zonas. Asimismo, la zona que más produce bienes agrícolas primarios, con el $63,27 \%$ del total de la producción, es la zona 5, seguida de la zona $7(12,76 \%)$ y la zona $1(10,45 \%)$.

El modelamiento de los datos permitió comprobar la hipótesis planteada en la primera sección de esta investigación, la cual manifestaba que el aumento en los niveles de crédito concedido, tanto público como privado, coadyuva al crecimiento de la producción agrícola. El modelo final (panel de efectos aleatorios) muestra que el crédito privado no explica el comportamiento de la producción agrícola, dado que su estimador es igual a cero (en términos estadísticos); por ende, cualquier interpretación o análisis respecto a la incidencia del crédito privado en la producción es espuria. En contraste, el crédito productivo público resultó tener una incidencia positiva y significativa, aunque bastante leve. En términos económicos, según lo arrojado en el modelamiento econométrico, la producción agrícola es poco sensible a los cambios que se efectúen en el crédito productivo. En adición, la delimitación por zonas de planificación mantiene una relevante significancia en los cambios que se dé en la producción, por lo cual es importante analizar los factores que emplean cada una de estas zonas. Esto permitirá extraer más variables que pueden tener algún impacto sobre el rendimiento productivo, $\mathrm{y}$ así darle mayor robustez al modelo y a las conclusiones que se lleguen.

En suma, el crédito productivo concedido hacia el sector agrícola genera una leve incidencia, casi nula, en el comportamiento de la producción de aquel sector; en otras palabras, la producción puede llegar a sostenerse sin la presencia del crédito productivo agrícola. Por esta razón, la hipótesis planteada no es aceptada.

\section{Recomendaciones}

En virtud de la importancia del sector agrícola en la economía ecuatoriana, se deberían crear políticas y estrategias participativas. Es decir, involucrar a los pequeños y medianos productores agrícolas en el diseño, formulación, ejecución y retroalimentación de las políticas públicas. Además, incluir 
al sector empresarial para la generación de estas, con la finalidad de crear sinergias entre las necesidades de cada sector.

Por otro lado, respecto a la política de expendio de créditos hacia el sector agrícola, como se observó, no poseen relevancia en el proceso de producción; se debería realizar una focalización, además de brindar un apoyo técnico y estratégico que tenga como finalidad maximizar los beneficios que se dan por la adquisición de un crédito. En adición, la innovación y desarrollo tecnológico en el agro ecuatoriano es una deuda que tiene Ecuador, y aquello es uno de los factores responsables de pérdida de competitividad frente a los países vecinos.

\section{Referencias}

Arellano, Manuel, y Olympia Bover. 1990. "La econometría de datos de panel”. Investigaciones Económicas (segunda época) 14 (1): 3-45. 〈http://bit.ly/2MHIcrH〉.

EC Instituto Nacional de Estadística y Censos (INEC). 2019. Ecuador en cifras. Consulta: mayo de 2019. 〈http://www.ecuadorencifras.gob.ec/estadisticas/〉.

EC Ministerio de Agricultura, Ganadería, Acuacultura y Pesca (MAGAP). 2019. Sistema de Información Pública Agropecuaria (SIPA). Consulta: mayo de 2019. «tttp://sipa.agricultura.gob.ec/s.

EC Secretaría Nacional de Planificación y Desarrollo (SENPLADES). 2019. Información de inversión pública. Quito: SENPLADES. Consulta: mayo de 2019. 〈http://www.planificacion.gob.ec/s.

EC Superintendencia de Bancos. 2019. Estudios y análisis. Consulta: mayo de 2019. 〈http:// bit.ly/2VNtAez).

Echavarría, Juan, Sara Restrepo, Mauricio Villamizar y Juan Hernández. 2017. Impacto del crédito sobre el agro en Colombia: evidencia del nuevo Censo Nacional Agropecuario. Bogotá: BID.

Fuentes, Alberto. 2005. "El impacto del crédito en la producción del sector agrícola en Venezuela, 1970-1999". Tesis de posgrado, Universidad de los Andes, Mérida. 〈http://bit. 1y/2OZy14T .

García Navarro, Clemente. 2017. "Las políticas de crédito al sector agropecuario en Nicaragua 1990-2012". Revista Científica Electrónica de Ciencias Humanas 12 (36): 24-44. 〈https://www.redalyc.org/pdf/709/70950101002.pdf〉.

Gujarati, Damodar, y Porter Dawn. 2010. Econometría. Ciudad de México: McGraw-Hill / Interamericana Editores S. A. 
Quinde Rosales, Francisco, Rina Bucaram Leverone y Victor Xavier Quinde Rosales. 2018. "Incidencia de la banca en el sector agrícola primario ecuatoriano". INNOVA Research Journal 3 (3): 53-61. Doi: 10.33890/innova.v3.n3.2018.421.

Viteri, María del Pilar, y Mauro Tapia. 2018. "Economía ecuatoriana: de la producción agrícola al servicio". Revista Espacios 39 (32): 30-36. 〈https://www.revistaespacios.com/ a18v39n32/a18v39n32p30.pdf .

Wooldridge, Jeffrey. 2010. Introducción a la econometría. Ciudad de México: Cengage Learning.

\section{Anexos}

\section{Resultados de los test entre los modelos de datos de panel}

\begin{tabular}{|l|c|c|c|l|}
\hline Comparación & \multicolumn{1}{|c|}{ Test } & Valor estadístico & Valor $\mathbf{P}$ & Decisión \\
\hline Pool vs. EF & Prueba F (1920) & 130,55 & $<2,2 \mathrm{e}-16$ & $\begin{array}{l}\text { Rechazo } \\
\text { Elección EF }\end{array}$ \\
\hline EA vs. Pool & $\begin{array}{l}\text { Breusch-Pagan } \\
(1979)\end{array}$ & 318,76 & $<2,2 \mathrm{e}-16$ & $\begin{array}{l}\text { Rechazo } \\
\text { Elección EA }\end{array}$ \\
\hline EF vs. EA & $\begin{array}{l}\text { Test Hausman } \\
(1978)\end{array}$ & 2,84 & 0,2413 & $\begin{array}{l}\text { Acepto } \\
\text { Elección EA }\end{array}$ \\
\hline
\end{tabular}

Nota. Con el apoyo del programa RStudio se logró comparar los modelos mediante los test estadísticos, teniendo como modelo final el tipo de Efectos Aleatorios (EA).

\section{Test para medir la dependencia transversal en paneles}

\begin{tabular}{|c|c|c|c|}
\hline Test & Valor estadístico & Valor $\mathbf{P}$ & Decisión \\
\hline Pesaran CD & 1,6376 & 0,1015 & No Cross-Sectional Dependence \\
\hline
\end{tabular}

Nota. Aplicando el comando en el programa RStudio se logró testear la dependencia transversal de las variables en paneles, donde la hipótesis nula es que no existe dependencia. Al tener un valor $p$ mayor a 0,05 , no existe evidencia estadística para rechazar la hipótesis nula. 


\section{Test para identificar homocedasticidad o heterocedasticidad}

\begin{tabular}{|c|c|c|c|}
\hline Test & Valor estadístico & Valor $\mathbf{P}$ & Decisión \\
\hline Breusch-Pagan & 9,9154 & 0,825 & Homocedasticidad \\
\hline
\end{tabular}

Nota. Aplicando un comando en el programa RStudio se logró testear la heterocedasticidad de los datos recopilados. La hipótesis nula para este test es que los datos tienen un comportamiento homocedástica, lo cual se pretende tener. Al arrojar un valor $p$ mayor a 0,05, no existe evidencia estadística para rechazar la hipótesis nula.

\section{Test para identificar correlación serial en el modelo de panel}

\begin{tabular}{|c|c|c|c|}
\hline Test & Valor estadístico & Valor $\mathbf{P}$ & Decisión \\
\hline Breusch-Godfrey/Wooldrige & 35.965 & 0,001 & $\begin{array}{c}\text { Correlación serial en los } \\
\text { errores idiosincrático }\end{array}$ \\
\hline
\end{tabular}

Nota. Aplicando un comando en el programa RStudio se logró testear la correlación serial en el modelo final de panel. La hipótesis nula para este test es que no existe correlación serial, pero al tener un valor $p$ inferior a 0,05 , existe evidencia estadística por lo cual no se acepta la hipótesis nula. Para corregir este error se crearon estimadores más consistentes/robustos. 